\title{
Effectiveness of Multilevel Botulinum Toxin A Injection with Integrated Treatment Program on Spasticity Reduction in Non-Ambulatory Young Children with Cerebral Palsy
}

\author{
Sebahat Aydila Fatma Merih Akpinar $^{\mathrm{a}} \quad$ Evren Akpinar $^{\mathrm{b}} \quad$ Kubilay Beng $^{\mathrm{b}}$ \\ Mehmet Firat Yagmurlub \\ a Physical Therapy and Rehabilitation, Gait Analysis Laboratory, Metin Sabanci Baltalimani Bone Diseases Education \\ and Research Hospital, Istanbul, Turkey; ${ }^{b}$ Metin Sabanci Baltalimani Bone Diseases Education and Research \\ Hospital, Pediatric Orthopaedics, Istanbul, Turkey
}

\section{Significance of the Study}

- The present study shows the effectiveness of Botulinum toxin A injection in non-ambulatory patients with cerebral palsy aged 4-8 years assessed at 3 different times after treatment. This intervention provides a possibility of delaying orthopedic surgeries for children whose parents do not want surgery for their young children and for patients who are at high risk for general anesthesia.

\section{Keywords}

Botulinum toxin - Cerebral palsy

\begin{abstract}
Objective: The aim of the present study was to evaluate the effectiveness of multilevel Botulinum Toxin A (BTX-A) injection, as part of an integrated approach, for the treatment of spasticity in non-ambulatory young children with diplegic cerebral palsy (CP). Subjects and Methods: Seventeen nonambulatory patients aged 4-8 years with diplegic CP (Gross Motor Function Classification System [GMFCS] level IV) were evaluated before and at $1 \mathrm{st}$, 3rd, and 6 th months after BTX-A injection. The effect of BTX-A on spasticity of gastrocnemius and hamstring muscles was assessed using the Modified Ashworth Scale (MAS) and Modified Tardieu Scale (MTS). The
\end{abstract}

velocity-dependent properties of spastic muscle as both slow (R2) and fast (R1) stretches were evaluated in MTS. Results: A statistically significant improvement was observed in $\mathrm{R} 1$ angles of gastrocnemius and hamstring muscles at $1 \mathrm{st}$ and 3rd months after BTX-A injection in non-ambulatory young children with CP. Statistically significant improvement was found in MAS of gastrocnemius and hamstring muscles and R2 angles of knee and ankle joint after 1st month of BTX-A injection. Conclusion: Multilevel BTX-A injection, as part of an integrated approach, can be used for focal treatment of spasticity, especially of hamstring and gastrocnemius muscles, in non-ambulatory young children with CP GMFCS level IV.

(c) 2019 The Author(s)

Published by S. Karger AG, Basel

\begin{tabular}{|c|c|c|}
\hline KARGER & $\begin{array}{l}\text { () } 2019 \text { The Author(s) } \\
\text { Published by S. Karger AG, Basel }\end{array}$ & $\begin{array}{l}\text { Karger } \\
\text { Open access }\end{array}$ \\
\hline $\begin{array}{l}\text { E-Mail karger@karger.com } \\
\text { www.karger.com/mpp }\end{array}$ & $\begin{array}{l}\text { This is an Open Access article licensed ur } \\
\text { Attribution-NonCommercial-4.0 Interna } \\
\text { (http://www.karger.com/Services/OpenA } \\
\text { the online version of the article only. Usa } \\
\text { mercial purposes requires written permi }\end{array}$ & $\begin{array}{l}\text { er the Creative Commons } \\
\text { onal License (CC BY-NC) } \\
\text { essLicense), applicable to } \\
\text { and distribution for com- } \\
\text { on. }\end{array}$ \\
\hline
\end{tabular}

Sebahat Aydil

Physical Therapy and Rehabilitation, Gait Analysis Laboratory

Metin Sabanci Baltalimani Bone Diseases Education and Research Hospital, 62 Sariyer TR-34470 Istanbul (Turkey)

E-Mail drsebahataydil@yahoo.com 


\section{Introduction}

Cerebral palsy $(\mathrm{CP})$ is a group of permanent disorders as a result of nonprogressive damage to the developing fetal or infant brain, which leads to the abnormal development of motor control and posture in children with $\mathrm{CP}$ [1]. Spasticity changes biomechanical properties of muscles and soft tissues around joints and may result in joint contractures, muscle shortening, pain, and functional limitations that negatively affect motor development in patients with CP [2].

Focal treatments are mainly represented by botulinum toxin A (BTX-A), which directly treats symptomatic muscles; when used at recommended doses, it avoids systemic side effects. The efficacy of BTX-A has been widely reported in the literature, particularly in the treatment of spasticity in ambulatory children with $\mathrm{CP}$. The most common indication of BTX-A in ambulatory children with spastic CP is management of gait dysfunctions, such as equinus gait or toe-walking by reducing muscle tone of the lower limbs [3, 4]. Children with severe forms of CP are non-ambulatory (Gross Motor Function Classification System [GMFCS] levels IV and V), unable to sit independently, and require significant assistance for daily activities. These children frequently have musculoskeletal pain or discomfort associated with severe spasticity, muscle contractures, and joint subluxation [5]. Despite a generalized pattern of spasticity, BTX-A injections also can be used in non-ambulatory young children with CP GMFCS level IV to improve posture and positioning, to ease the use of orthoses and wheel chair, to facilitate personal care, and to enhance compliance to physical therapy [6].

To prevent the development of secondary problems like soft tissue contractures and skeletal deformities, a comprehensive and combined approach to treatment, including both pharmacological and rehabilitative interventions, is needed in these children. An integrated approach including orthotic management, casting and physiotherapy is of major importance for the maximum benefit of BTX-A injections. This approach might delay or decrease the need for surgical interventions especially in young children, while reserving single-event, multilevel surgery for fixed musculotendinous contractures and bony deformities in older children [7].

The aim of this study was to investigate the efficacy of multilevel injection of BTX-A in a single session in the treatment of spasticity in non-ambulatory young children with CP GMFCS level IV. We hypothesized that multilevel BTX-A injections with casting, orthoses, and intensive physiotherapy would decrease spasticity and increase the range of motion (ROM) in the lower extremities in non-ambulatory young children with CP.

\section{Subjects and Methods}

\section{Subjects}

Non-ambulatory children with spastic $\mathrm{CP}$ who met the inclusion criteria were enrolled for this study. Inclusion criteria included spastic diplegia with no history of BTX-A treatment within 6 months, no intrathecal baclofen or oral medication usage, no orthopedic surgery or selective dorsal rhizotomy, aged between 4 and 8 years, spasticity in 2 or more lower extremity muscle groups that interferes with personal care and use of orthotic devices, and not able to walk independently. Exclusion criteria were as follows: fixed contractures of lower extremities, BTX-A injection within 6 months, intrathecal baclofen or oral medication usage during the postinjection period and follow-up visit, contraindication for BTX-A injections or general anesthesia, and not being able to follow-up for 6 months after the procedure. Seventeen non-ambulatory patients ( 6 girls, 11 boys) with spastic diplegic CP with GMFSC level IV were enrolled in this study. Their mean age at the time of the applications was 5 years (range $4-8$ years).

\section{Procedures}

BTX-A injections were applied under mask anesthesia as part of the integrated treatment approach previously described by Molenaers et al. [7]. The muscles selected for treatment were injected at multiple sites with a maximum dose of $50 \mathrm{IU} /$ muscle and maximum total dose $20 \mathrm{IU} / \mathrm{kg}$. The dose of BTX-A for each muscle was based on the size of the muscles, degree of spasticity and patients' weight [8]. The injection sites were determined by palpation of muscle belly and needle placement was verified either by stretching the muscle or by moving the distal part of the joints through a specific ROM to observe motion of the needle placed in the target muscle [9]. The BTX-A was applied to gastrocnemius and hamstring muscles in all treated extremities. Other than these muscles, BTX-A was also applied to the psoas muscle in 20 extremities, to rectus femoris in 14 extremities, to soleus in 10 extremities, and to adductors in 20 extremities.

After BTX-A injections, stretching plaster casts were applied up to the proximal parts of the lower extremities with knee in extension and ankle joint in neutral position for 10 days. After removal of stretching casts, all patients had solid ankle foot orthoses and knee extension braces, and received routine physiotherapy consisting of passive stretching of flexor and adductor muscles and strengthening of extensor muscles, as part of the integrated approach. The patients received physiotherapy 3 times a week for 12 weeks, and each physiotherapy session lasted for about $60 \mathrm{~min}$ [7, 10-12].

\section{Clinical Measurement of Spasticity}

The patients were evaluated before and at 1st, 3rd, and 6th months after BTX-A injections. The spasticity of psoas muscle was assessed with the Thomas test and spasticity of rectus femoris was evaluated with the Duncan Ely test. The effect of BTX-A on spasticity of gastrocnemius and hamstrings was assessed by the Modified Ashworth Scale (MAS) and the Modified Tardieu Scale (MTS) 
Table 1. Changes in spasticity of gastrocnemius and hamstring muscles with MAS and MTS

\begin{tabular}{|c|c|c|c|c|c|}
\hline Parameters & Pre BTX-A & $\begin{array}{l}\text { Post BTX-A } \\
1 \text { st month }\end{array}$ & $\begin{array}{l}\text { Post BTX-A } \\
\text { 3rd month }\end{array}$ & $\begin{array}{l}\text { Post BTX-A } \\
6 \text { th month }\end{array}$ & $p$ \\
\hline MAS $^{a}$ of gastrocnemius muscle & $2(1-3)$ & $1(0-3)$ & $1(0-3)$ & $2(0-3)$ & $0.000^{*}$ \\
\hline $\mathrm{R} 1$ of $\mathrm{MTS}^{\mathrm{b}}$ of ankle $\mathrm{c}^{\mathrm{c}}$ & $-14 \pm 15$ & $-2 \pm 13$ & $-6 \pm 13$ & $-10 \pm 14$ & $0.000^{* *}$ \\
\hline $\mathrm{R} 2$ of $\mathrm{MTS}^{\mathrm{b}}$ of ankle $\mathrm{e}^{\mathrm{c}}$ & $-1 \pm 15$ & $5 \pm 11$ & $4 \pm 11$ & $0 \pm 15$ & $0.006^{*}$ \\
\hline $\mathrm{R} 2-\mathrm{R} 1$ of $\mathrm{MTS}^{\mathrm{b}}$ of ankle $\mathrm{c}^{\mathrm{c}}$ & $12 \pm 10$ & $8 \pm 5$ & $10 \pm 6$ & $10 \pm 7$ & $0.16^{*}$ \\
\hline MAS $^{a}$ of hamstring muscles & $2(1-3)$ & $1(0-2)$ & $1(0-3)$ & $2(0-3)$ & $0.000^{*}$ \\
\hline R1 of MTS $^{\mathrm{b}}$ of popliteal angle & $67 \pm 7$ & $55 \pm 11$ & $59 \pm 13$ & $63 \pm 12$ & $0.000^{* *}$ \\
\hline R2 of $\mathrm{MTS}^{\mathrm{b}}$ of popliteal angle & $59 \pm 8$ & $53 \pm 9$ & $54 \pm 9$ & $58 \pm 9$ & $0.002^{* *}$ \\
\hline R2-R1 of MTS ${ }^{b}$ of popliteal angle & $-8 \pm 7$ & $-2 \pm 7$ & $-5 \pm 7$ & $-5 \pm 5$ & $0.002^{*}$ \\
\hline
\end{tabular}

[13]. The velocity-dependent properties of spastic muscle as both slow and fast stretches were evaluated in MTS [14]. Two levels of the ankle dorsiflexion and popliteal angles were measured after slow and fast stretches, referring to R2 and R1 angles respectively [15]. The relationship between the fast (R1) and slow (R2) angles was evaluated. The neutral position of the ankle joint was zero degrees, the values measured in ankle dorsiflexion were positive, while the values measured in ankle plantar flexion were negative.

The study was approved by the Institutional Review Board of our hospital. Parents of the study subjects provided written informed consent.

\section{Statistical Analysis}

Mean, median, maximum, minimum, and standard deviation values were used in descriptive statistics of the data. The data distribution was checked with the Kolmogorov-Simirnov test. To define the effects of BTX-A on spasticity of muscles; repeated measures analysis of variance (ANOVA) and least significant difference (LSD) were used for pairwise comparison of parametric data, and Friedman and Wilcoxon tests were used for pairwise comparison of non-parametric data. The significance level for ANOVA and Friedman test was $p<0.05$. Pairwise comparisons using LSD and Wilcoxon tests were subject to the Bonferroni correction, for these analyses the significance level was $p<0.008$. SPSS statistical program (version 22.0; IBM, Armonk, NY, USA) was used for statistical analyses.

\section{Results}

There was a significant one grade of improvement in MAS of hamstring muscles after BTX-A injection at the 1 st month and this effect on spasticity continued at the $3 \mathrm{rd}$ month of injection, but it was not statistically significant (Wilcoxon test, Bonferroni correction; pre-post BTX-A 1 st month $p=0.000$, pre-post BTX-A 3rd month $p=0.012$, respectively). The spasticity of hamstrings increased at the 6 th month of therapy and returned to baseline value (Wil- coxon test, Bonferroni correction; pre-post BTX-A 6th month $p=0.253$ ). Statistically significant improvement was found in the R1, R2, and R2-R1 angles of knee joint after BTX-A injection (ANOVA test $p=0.000, p=0.002$, $p=0.002$, respectively). The LSD test revealed that the mean R2 angle of knee joint significantly decreased at 1st month and this improvement continued at the $3 \mathrm{rd}$ month of injection, but it was not statistically significant after Bonferroni correction (pre-post BTX-A 1st month, $p=$ 0.002 , post-BTX-A 1st-post BTX-A 3rd month, $p=0.028$ ). At the end of the 6th month, when compared with baseline values, the recoveries in mean R2 angle of knee joint were lost (pre-post BTX-A 6th month, $p=0.499$ ). The mean R1 angle of knee joint significantly decreased after therapy (ANOVA test $p=0.000$ ). The LSD test revealed that there was statistically significant improvement in the mean R1 angle of knee joint at 1st and 3rd months of therapy (pre-post BTX-A 1st month $p=0.000$, pre-post BTXA 3rd month $p=0.000$ ). Although this improvement in mean R1 angle continued at the 6th month and did not return to baseline, it was not significant after Bonferroni correction (pre-post BTX-A 6th month $p=0.04$ ). There was a significant change in mean R2-R1 angle of knee joint after BTX-A injection (Friedman test $p=0.002$ ). Post hoc analysis revealed that a statistically significant decrease was found in the mean R2-R1 angle at the 1st month of therapy; although this effect deteriorated at the 3rd month, it did not return to baseline at the end of the 6th month (Wilcoxon test, Bonferroni correction; pre-post BTX-A 1st month $p=0.001$ pre-post BTX-A 3rd month $p=0.066$ pre-post BTX-A 6th month $p=0.066$; Table 1 ).

A statistically significant one grade improvement in MAS of the gastrocnemius muscle was observed at 1st month after therapy, and this effect on spasticity contin- 
Table 2. Clinical examination data

\begin{tabular}{|c|c|c|c|c|c|}
\hline & $\begin{array}{l}\text { Extremities, } \\
n\end{array}$ & Mean \pm SD & Median & Range & $p$ value \\
\hline Thomas test $^{\mathrm{a}}$ & 20 & & & & $0.01^{*}$ \\
\hline Before BTX-A injection & & $15 \pm 7$ & 15 & $0-25$ & \\
\hline After 1st month of the injection & & $13 \pm 9$ & 15 & $0-30$ & \\
\hline After 3rd month of the injection & & $18 \pm 11$ & 20 & $0-35$ & \\
\hline After 6th month of the injection & & $17 \pm 11$ & 20 & $0-35$ & \\
\hline Hip abduction degrees & 20 & & & & $0.28^{*}$ \\
\hline Before BTX-A injection & & $47 \pm 8$ & 50 & $20-60$ & \\
\hline After 1st month of the injection & & $49 \pm 7$ & 50 & $35-65$ & \\
\hline After 3rd month of the injection & & $49 \pm 10$ & 50 & $25-70$ & \\
\hline After 6th month of the injection & & $48 \pm 10$ & 50 & $25-65$ & \\
\hline
\end{tabular}

Table 3. Rectus femoris spasticity

\begin{tabular}{lllll}
\hline & $\begin{array}{c}\text { Extremi- } \\
\text { ties, } n\end{array}$ & Mean & Range $p$ value \\
& 14 & & & $0.17^{*}$ \\
Before BTX-A injection & & 1 & $0-3$ & \\
After 1st month of the injection & 0 & $0-3$ & \\
After 3rd month of the injection & 0 & $0-3$ & \\
After 6th month of the injection & 1 & $0-3$ &
\end{tabular}

${ }^{*} p=$ Friedman test.

ued at the 3rd month of BTX-A injection (Wilcoxon test, Bonferroni correction; pre-post BTX-A 1st month $p=$ 0.000 , pre-post BTX-A 3rd month $p=0.008)$, but deteriorated at the 6th month of therapy (pre-post BTX-A 6th month $p=0.643$ ). The mean of $\mathrm{R} 1$ angle of the ankle joint with knee extension significantly improved at the 1st month of BTX-A injection and this improvement continued at the 3rd month of therapy (LSD test) (pre-post BTX-A 1st month $p=0.000$, pre-post BTX-A 3rd month $p=0.000$ ), and recoveries were lost when compared with baseline at the 6th month (pre-post BTX-A 6th month, $p=0.064$ ). Statistically significant improvement was found in the mean R2 angle of ankle joint with knee extension after therapy (Friedman test, $p=0.006$ ). Post hoc analysis test revealed that there was a significant improvement in the mean R2 angle of the ankle at the 1st month, and this effect continued until the 3rd month of therapy, but it was not statistically significant (Wilcoxon test, Bonferroni correction; pre-post BTX-A 1st month $p=0.003$ pre-post BTX-A 3rd month $p=0.016$ ). At the end of the 6th month, the effect of BTX-A on the R2 angle of ankle joint was lost (pre-post BTX-A 6st month $p=0.707$ ). There was no significant difference in the mean R2-R1 angle of MTS of the ankle joint with knee extension after BTX-A injection (Friedman test $p=0.167$ ).

When the effect of BTX-A on the spasticity of hip flexors of 20 extremities was evaluated with the Thomas test, statistically significant changes were found (Friedman test $p=$ 0.021). The Wilcoxon signed ranks test revealed that there were improvements in the Thomas test values at the 1st month of therapy when compared to baseline, but it was not statistically significant (pre-post BTX-A 1st month $p=$ 0.318). After that, the Thomas test values deteriorated significantly at the 3rd and 6th months compared to values at the 1st month of therapy (Wilcoxon signed ranks test, Bonferroni correction; post BTX-A 1st-post BTX-A 3rd month $p=0.003$, post BTX-A 3rd - post BTX-A 6th month $p=$ $0.495)$. There was no significant change in measurement of hip abduction in patients (20 extremities) who had BTX-A injection to hip adductors (Friedman test $p=0.336$; Table 2 ). There was no significant change in terms of rectus femoris spasticity (Friedman test $p=0.167$; Table 3 ).

\section{Discussion}

The present study showed that there was a statistically significant improvement on mean R1 angles of gastrocnemius and hamstring muscles at 1st and 3rd months after BTX-A injection in non-ambulatory young children with CP GMFCS level IV. Statistically significant improvement was found in the MAS, R1, and R2 angles of the knee and ankle joints after the 1st month of BTX-A injection. 
Despite a generalized pattern and severity of spasticity, BTX-A injections can be used in non-ambulatory young children with CP GMFCS level IV to improve posture and positioning, to ease the use of orthoses and wheel chair, to facilitate personal care and to enhance compliance to physical therapy [6]. Early treatment of spasticity might decrease the need for orthopedic surgeries especially in young children, while reserving single-event, multi-level surgery for fixed musculotendinous contractures and bony deformities in older children $[7,9]$. The present study reveals that multilevel BTX-A injection is effective in the treatment of spasticity in non-ambulatory young children with CP GMFCS level IV, and might help delay the need for orthopedic surgeries.

The relationship between the $\mathrm{R} 1$ and $\mathrm{R} 2$ angles estimates the relative contributions of spasticity compared to contracture. Slow velocity examination of R2 through passive joint ROM indicates that muscle length was controlled by neural and non-neural components [13]. Boyd et al. [16] found a larger value of $\mathrm{R} 2-\mathrm{R} 1$, and indicates the more $\mathrm{dy}$ namic component, which is more likely to respond to BTX$\mathrm{A}$ injection. Lin et al. [17] found that R2 and R2-R1 angles of the gastrocnemius muscles improved significantly after the 1st month of BTX-A injection. In the present study, the assessment of R2 and R2-R1 angles of hamstrings statistically significant improvement at the 1st month after BTX-A injection. Significant improvement was noted on mean R1 angles of gastrocnemius and hamstrings muscles at the 1st and 3rd months after BTX-A injection. It means that the neural component of spasticity improved at the 1st month and this effect continued at the 3rd month of therapy. The improvement on mean R1 angles of gastrocnemius and hamstring muscle was lost after the 6th month.

MAS is the most common clinical scale used to assess spasticity. Despite its widespread clinical use, the reliability of this scale has been questioned in some studies [18]. MTS is another clinical scale used to assess spasticity; it is not as common as MAS but has been proposed as a more effective method in assessing spasticity $[18,19]$. In the present study, based on MTS measurements, spasticity of the gastrocnemius and hamstrings muscles showed significant improvement at the 1st month and this effect continued at 3 months after the BTX-A injection.

Another aspect of the present study is that patients in this study were aged 4-8 years. Previous studies suggest that superior treatment outcomes were obtained with a treatment at earlier ages. Linder et al. [20] reported that higher functional outcomes were seen for younger (age $<5$ years) and moderately impaired children with GMFCS III level. Boyd et al. [21] emphasized that BTX-A treat-

BTX Injection in Non-Ambulatory CP Patients ment should be done within the age range of $1-5$ years in order to gain optimal outcomes. In the present study, significant improvement was seen in the spasticity of hamstring and gastrocnemius muscles in non-ambulatory children with CP GMFCS level IV even they are in the age range of 4-8 years. We believe that this is important for parents who do not want surgery for their children at earlier ages, and also for families whose children are at high risk for general anesthesia. Additionally, BTX-A treatment might increase compliance to physical therapy and ease the use of orthoses in these patients and it can satisfy the expectations of their parents.

The BTX-A injection of hip adductors is used in more severely involved children with GMFCS level IV-V to improve positioning, to ease personal care or to prevent progressive hip displacement [22, 23]. Mall et al. [24] stated that there was significant improvement in the spasticity of the adductor muscles in children (mean age 6 years) with CP GMFCS level I-V at 1st month, and that this improvement continued until the 3rd month after BTX-A injection. Although there was no significant improvement in the spasticity of hip adductors in our study, BTXA injection was applied to hip adductors only in 20 extremities and not to all extremities.

Scholtes et al. [25] evaluated the effect of multilevel BTXA injections on gait pattern and spasticity as part of an integrated therapy in 46 children with spastic CP. They found that there was a significant improvement in the spasticity of hamstrings, soleus, and gastrocnemius muscles after 6 weeks of injections, which was maintained at 12 weeks but not at 24 weeks after treatment. While the patients were ambulatory with GMFCS level I-III in their study, the patients who were non-ambulatory with GMFCS level IV showed significant improvement on spasticity in the present study. We believe that these findings are important to demonstrate the effectiveness of the BTX-A injection in non-ambulatory young children with CP GMFCS level IV.

All patients in our study received the integrated therapy, which consisted of casting, orthoses and intensive physiotherapy after BTX-A injection. Therefore, our findings of improvement in the spasticity of hamstring and gastrocnemius muscles are compatible with the results of previous studies. The total effect, rather than the contribution of each individual component, of the integrated treatment program was evaluated in this study. Therefore, we do not know how much of the effect is specifically attributable to the multilevel BTX-A injections alone. This is one of the limitations of the present study. Other limitations include the fact that the improvement on personal care, dressing, and positioning was not as- 
sessed with any outcome measures and also we could not assess the burden of care of these patients on their parents or caregivers before and after BTX-A treatment.

\section{Conclusion}

The present study shows the effectiveness of BTX-A injection in a non-ambulatory CP patients aged between 4 and 8 years with GMFCS level IV by assessments done at 3 different times after treatment. It demonstrates that multilevel BTX-A injection as part of the integrated approach can be used for focal treatment of spasticity especially of hamstring and gastrocnemius muscles in nonambulatory young children with CP GMFCS level IV. Such a treatment affords the possibility of delaying orthopedic surgeries for children whose parents do not want surgery for their young children, and for patients who are at high risk for general anesthesia.

\section{References}

1 Rosenbaum P, Paneth N, Leviton A, Goldstein M, Bax M, Damiano D, et al. A report: the definition and classification of cerebral palsy April 2006. Dev Med Child Neurol Suppl. 2007 Feb;109:8-14.

2 Dietz V, Sinkjaer T. Spastic movement disorder: impaired reflex function and altered muscle mechanics. Lancet Neurol. 2007 Aug; 6(8):725-33.

3 Koog YH, Min BI. Effects of botulinum toxin A on calf muscles in children with cerebral palsy: a systematic review. Clin Rehabil. 2010 Aug;24(8):685-700.

4 Ryll U, Bastiaenen C, De Bie R, Staal B. Effects of leg muscle botulinum toxin A injections on walking in children with spasticity-related cerebral palsy: a systematic review. Dev Med Child Neurol. 2011 Mar;53(3):210-6.

5 Ramstad K, Jahnsen R, Skjeldal OH, Diseth TH. Characteristics of recurrent musculoskeletal pain in children with cerebral palsy aged 8 to 18 years. Dev Med Child Neurol. 2011 Nov;53(11):1013-8.

6 Love SC, Novak I, Kentish M, Desloovere K, Heinen F, Molenaers G, et al.; Cerebral Palsy Institute. Botulinum toxin assessment, intervention and after-care for lower limb spasticity in children with cerebral palsy: international consensus statement. Eur J Neurol. 2010 Aug;17 Suppl 2:9-37.

7 Molenaers G, Desloovere K, Eyssen M, Decaf J, Jonkers I, Cock PD. Botulinum toxin type A treatment of cerebral palsy: an integrated approach. Eur J Neurol. 1999;6:s51-7.

8 Molenaers G, Van Campenhout A, Fagard K, De Cat J, Desloovere K. The use of botulinum toxin $\mathrm{A}$ in children with cerebral palsy, with a focus on the lower limb. J Child Orthop. 2010 Jun;4(3):183-95.

9 Van Campenhout A, Molenaers G. Localization of the motor endplate zone in human skeletal muscles of the lower limb: anatomical guidelines for injection with botulinum toxin. Dev Med Child Neurol. 2011 Feb;53(2):10819.
10 Scholtes VA, Dallmeijer AJ, Knol DL, Speth LA, Maathuis CG, Jongerius PH, et al. The combined effect of lower-limb multilevel botulinum toxin type a and comprehensive rehabilitation on mobility in children with cerebral palsy: a randomized clinical trial. Arch Phys Med Rehabil. 2006 Dec;87(12):1551-8.

11 Hawamdeh ZM, Ibrahim AI, Al-Qudah AA. Long-term effect of botulinum toxin (A) in the management of calf spasticity in children with diplegic cerebral palsy. Eura Medicophys. 2007 Sep;43(3):311-8.

12 Desloovere K, Molenaers G, Jonkers I, De Cat J, De Borre L, Nijs J, et al. A randomized study of combined botulinum toxin type $\mathrm{A}$ and casting in the ambulant child with cerebral palsy using objective outcome measures. Eur J Neurol. 2001 Nov;8(s5 Suppl 5):75-87.

13 Boyd RN, Graham HK. Objective measurement of clinical findings in the use of botulinum toxin type a for the management of children with cerebral palsy. Eur J Neurol. 1999; 6:s23-35.

14 Patrick E, Ada L. The Tardieu Scale differentiates contracture from spasticity whereas the Ashworth Scale is confounded by it. Clin Rehabil. 2006 Feb;20(2):173-82.

15 Yam WK, Leung MS. Interrater reliability of Modified Ashworth Scale and Modified Tardieu Scale in children with spastic cerebral palsy. J Child Neurol. 2006 Dec;21(12):10315.

16 Boyd R, Graham HK. Botulinum Toxin A in the management of children with cerebral palsy: indications and outcome. Eur J Neurol. 1997;4:15-22.

17 Lin YC, Lin IL, Chou TF, Lee HM. Quantitative evaluation for spasticity of calf muscle after botulinum toxin injection in patients with cerebral palsy: a pilot study. J Neuroeng Rehabil. 2016 Mar;13(1):25.

18 Alhusaini AA, Dean CM, Crosbie J, Shepherd $\mathrm{RB}$, Lewis J. Evaluation of spasticity in children with cerebral palsy using Ashworth and Tardieu Scales compared with laboratory measures. J Child Neurol. 2010 Oct;25(10): 1242-7.
19 Fosang AL, Galea MP, McCoy AT, Reddihough DS, Story I. Measures of muscle and joint performance in the lower limb of children with cerebral palsy. Dev Med Child Neurol. 2003 Oct;45(10):664-70.

20 Linder M, Schindler G, Michaelis U, Stein S, Kirschner J, Mall V, et al. Medium-term functional benefits in children with cerebral palsy treated with botulinum toxin type A: 1-year follow-up using gross motor function measure. Eur J Neurol. 2001 Nov;8(s5 Suppl 5): 120-6.

21 Boyd RN, Graham JE, Nattrass GR, Graham HK. Medium-term response characterisation and risk factor analysis of botulinum toxin type $\mathrm{A}$ in the management of spasticity in children with cerebral palsy. Eur J Neurol. 1999;6:s37-45.

22 Boyd RN, Dobson F, Parrott J, Love S, Oates J, Larson A, et al. The effect of botulinum toxin type A and a variable hip abduction orthosis on gross motor function: a randomized controlled trial. Eur J Neurol. 2001 Nov;8(s5 Suppl 5):109-19.

23 Graham HK, Boyd R, Carlin JB, Dobson F, Lowe K, Nattrass G, et al. Does botulinum toxin a combined with bracing prevent hip displacement in children with cerebral palsy and "hips at risk"? A randomized, controlled trial. J Bone Joint Surg. 2008;90(1):23-33.

24 Mall V, Heinen F, Siebel A, Bertram C, Hafkemeyer $\mathrm{U}$, Wissel J, et al. Treatment of adductor spasticity with BTX-A in children with $\mathrm{CP}$ : a randomized, double-blind, placebocontrolled study. Dev Med Child Neurol. 2006 Jan;48(1):10-3.

25 Scholtes VA, Dallmeijer AJ, Knol DL, Speth LA, Maathuis CG, Jongerius PH, et al. Effect of multilevel botulinum toxin a and comprehensive rehabilitation on gait in cerebral palsy. Pediatr Neurol. 2007 Jan;36(1):30-9. 